\title{
Generating Smooth Surfaces by Refinement of Curves
}

\author{
Uri Itai \\ Department of Mathematics, Technion, Haifa, Israel \\ ${ }^{*}$ Corresponding Author: uritai@ fermat.technion.ac.il
}

Copyright (c)2014 Horizon Research Publishing All rights reserved.

\begin{abstract}
Generalization of subdivision schemes refining points to schemes refining more complex geometric objects has become popular in recent years. In this paper we generalize corner-cutting schemes in order to refine curves taking into account the geometry of the curves. We provide conditions guaranteeing that these schemes are well defined and converge to a surfaces with contentious tangents.
\end{abstract}

Keywords Subidivision, Refinement, Curves, Surfaces

\section{Introduction}

Subdivision schemes are attractive methods for generating a smooth object from discrete data by repeated refinements. These schemes have many desirable properties such as simplicity, fast convergence and smoothness of the generated objects. Therefore, subdivision schemes have gained popularity in recent years as an important tool in approximation, in computer graphics and in computer aided geometric design [15, 2].

The most popular non-linear subdivision schemes are what we term asymptotic-linear schemes, namely schemes whose behaviour is similar to that of "linear schemes" for sufficiently dense data. Originally subdivision schemes were designed to refine data consisting of points in a Euclidean space. Later these schemes were adapted to manifold-valued data such as Lie groups (for example see Grohs and Wallner [12]). All these schemes provide approximation to manifold-valued functions whenever the data consists of samples of such afunction.

The most basic non-trivial subdivision schemes refining points are the corner cutting schemes [14, 8]. A corner cutting scheme generates a sequence of refined points $\left\{\bar{p}_{i}\right\}_{i=0}^{2 n-1}$ from a given sequence of point $\left\{p_{i}\right\}_{i=0}^{n}$ according to the refinement rule,

$$
\bar{p}_{2 i}=(1-\mu) p_{i}+\mu p_{i+1}, \quad \bar{p}_{2 i+1}=\mu p_{i}+(1-\mu) p_{i+1}, \quad i=0,1, \ldots, n-1,
$$

where $0<\mu<\frac{1}{2}$, is called the refinement parameter.

Let us denote by $\mathcal{S}$ the refinement step operator that generates $\left\{\bar{p}_{i}\right\}_{i=0}^{2 n+1}$ from $\left\{p_{i}\right\}_{i=0}^{n}$. The repeated application of the refinement operator produces a sequence of point sequences $\left\{p_{i}^{j}\right\}_{i=0}^{n_{j}}, j=0,1, \ldots$, with $\left\{p_{i}^{0}\right\}_{i=0}^{n_{0}}$ the given initial points and $n_{j}=2^{j}\left(n_{0}-1\right)+1$. We define the corresponding polylines $\left\{\mathfrak{L}_{j}\right\}_{j=0}^{\infty}$, where $\mathfrak{L}_{j}=\mathfrak{L}\left(\left\{p_{i}^{j}\right\}_{i=0}^{n_{j}}\right)$ is the polyline through the refined points $\left\{p_{i}^{j}\right\}_{i=0}^{n_{j}}$. This sequence of polylines converges to a smooth limit curve [11], even when the refinement parameter $\mu$ depends on $j$ and $i$. The special case when $\mu=\frac{1}{4}$, is the Chaikin scheme [3], and its limit is

$$
C(t)=\sum_{i=0}^{n_{0}} p_{i}^{0} B_{2}(t-i), \quad \frac{1}{2} \leq t \leq n_{0}-\frac{1}{2},
$$

where $B_{2}(t)$ is a quadratic B-spline with integer knots and support $[0,3]$ (e.g., see [5, 9]).

The generalization of the scheme from points to a family of non-intersecting 3D curves is interesting both from the mathematical point of view and from the application aspects. However, these generalizations are not well defined for all initial curves even when using a linear scheme, which for any parametrization of the given curves the corner cutting scheme to each polyline consisting of all the points on the curves with the same parameter value. In this paper we introduce conditions that ensure the existence of a "smooth" limit surface to the generalized schemes.

A different extension to curves of the corner cutting schemes was done by Conti and Dyn [7], where the refinement operates on nets of intersecting curves ("Cartesian grids of curves"). They suggest a linear scheme that is based on the parametrization of the initial curves. Their scheme converges to smooth surfaces even when the curves are only piecewise smooth. 
This work is a second attempt of the author to generalize the corner cutting scheme to 3D non-intersecting curves. The first attempt (joint work with Dyn [13]) is purely geometrical and the generated limits of the scheme are not necessarily smooth surfaces, but only proved to be 3D sets which retain important properties of the initial curves. Most of the results obtained for the previous attempt hold here. However, in the present attempt we show that the generated limits of the scheme are surfaces, with Hölder continuous one side tangent, whenever the initial curves have a smooth arc-length parametrization and satisfy several additional conditions.

This paper is arranged as follows: We start in Section 2 by defining curves and correspondences. In Section 3 we define the family of "facet cutting" schemes. In Section 4 we investigate the "piecewise ruled surfaces", interpolating the refined curves at each refinement level. The "regularity" of the generated "piecewise ruled surfaces" is done in Section 5. In Section 6 we show some of the properties of a single refinement step. Next, in Section 7 the repeated refinement step is investigate. Convergence is proved in Section 8. In Section 9 we proved the the limit set is a surface and study its parametrization. Additional properties of the generated curves is given in Section 10. Auxiliary results and involved proofs are are deferred to the Appendix.

\section{Curves and Correspondences}

First we define the two fundamental objects on which the scheme performs "curves" and "correspondence". The initial curves take the place of the control points in point refining schemes. The structure of curves is much more complex than that of control points. Therefore, we restrict our investigation to the following cases.

Assumption 2.1. All the initial curves are contained in a compact set K. Moreover, each of the initial curves consists of a single connected component which is either closed in all the curves or has two end-points in all the curves.

We call curves with the last property curves of the same topology. We use the notation of do Carmo ([10], Chapter 1) for defining the curves. Let $C$ be a parametrized curve with parameter $t$, i.e.,

$$
C=\{(x(t), y(t), z(t)), \quad t \in I(C)\},
$$

where $I(C)$ is a closed interval in $\mathbb{R}$ which is called the parametric interval of the curve $C$ with respect to the parametrization $t$. The set of all the curves with first derivative $\frac{d}{d t} C(t)=\left(\frac{d}{d t} x(t), \frac{d}{d t} y(t), \frac{d}{d t} z(t)\right)$ which is continuous for all $t \in I(C)$ is denoted by $\mathbf{C}^{1}(I)$.

Definition 2.2. We call a curve $C \in \mathbf{C}^{1}(I)$ a smooth curve if there exist constants $0<\alpha \leq 1$ and $0<\mathrm{H}_{\text {tan }}(C)<\infty$ such that

$$
\left\|C(\tilde{t})-C(t)-\frac{d}{d t} C(t)(\tilde{t}-t)\right\| \leq \mathrm{H}_{t a n}(C)|t-\tilde{t}|^{1+\alpha}, \quad \forall t, \tilde{t} \in I(C) .
$$

The constant $\alpha$ is called the Hölder exponent of the curve $C$, and $\mathrm{H}_{\text {tan }}(C)$ is called the Hölder constant of the curve $C$.

Hereafter, we assume that all the initial curves are smooth. To avoid degenerate cases we assume that its derivative of each initial curve does not vanish at all the points.

If we have $\left\|\frac{d}{d t} C(t)\right\|=1$ for every $t \in I(C)$ then $t$ is the arc length parametrization of the curve $C$. In this case $\frac{d}{d t} C(t)$ is denoted by $\dot{C}(t)$.

Unless otherwise stated, we denote by $I(C)$ the parametric interval of a curve $C$, parametrized by arc length. We note that the curve are not necessary of the same length.

We denote by $\mathrm{H}_{t a n}(C)$ the minimal constant satisfying (2.1). The intermediate value theorem implies that (2.1) is equivalent to

$$
\left\|\frac{d}{d t} C(t)-\frac{d}{d t} C(\tilde{t})\right\| \leq \mathrm{H}_{t a n}(C)|t-\tilde{t}|^{\alpha}, \quad t, \tilde{t} \in I(C)
$$

In [8] deBoor showed that for a fixed $\mu$ in (1.1) there exists a Hölder exponent $\alpha$ and a Hölder constant $\mathrm{H}_{t a n}$ such that the limit curves of the corner cutting scheme satisfy (2.1) ${ }^{1}$. Both constants $\alpha$ and $\mathrm{H}_{\text {tan }}$ depend only on the refinement parameter $\mu$.

To define a curves" "refinement operator" for curves we need the next definition.

Definition 2.3. Let $C(t)$ and $\widetilde{C}(\widetilde{t})$ be two curves with parametrizations $t$ and $\tilde{t}$ defined on the parametric intervals $I(C)$ and $\widetilde{I}(\widetilde{C})$ respectively. A bijective mapping $v(t): I(C) \rightarrow I(\tilde{C})$ that satisfies:

1. The derivative $\frac{d}{d t} v$ exist and is positive.

2. The derivative is Hölder continuous,

$$
\left|\frac{d}{d t} v\left(t_{1}\right)-\frac{d}{d t} v\left(t_{2}\right)\right|<H_{v}\left|t_{1}-t_{2}\right|^{\alpha}
$$

3. $\frac{d}{d t} v$ is bounded away from zero.

\footnotetext{
${ }^{1}$ In the Chaikin scheme $\left(\mu=\frac{1}{4}\right)$ we get the Liptchitz condition $(\alpha=1$ in (2.1)).
} 
is called a smooth correspondence between $C(t)$ and $\widetilde{C}(\widetilde{t})$.

In this paper we consider only smooth correspondences and refer to them as correspondences although the term "correspondence" was defied previously in [13] in a slightly different way.

Since each correspondence between $C(t)$ and $\widetilde{C}(\widetilde{t})$ is bijective and its derivative is continuous and bounded away from zero, the inverse map is a correspondence between the curves $\widetilde{C}(\widetilde{t})$ and $C(t)$.

By using the fact that the curves are contained in a compact set in $\mathbb{R}^{3}$, one can show that the constant $\mathrm{H}_{\text {tan }}(C)$ of $(2.1)$ is invariant under any re-parametrization.

If $C(t)$ and $\widetilde{C}(\widetilde{t})$ have the same topology then any correspondence between $I(C)$ and $I(\widetilde{C})$ defines a bijection between the curves. However, this holds also the other way around. Every bijective smooth function between the curves $C(t)$ and $\widetilde{C}(\widetilde{t})$ induces a correspondence between the parametrization intervals $I(C)$ and $I(\widetilde{C})$.

Examples of correspondences can be found in $[6,4]$.

To avoid degenerate cases we further assume,

Assumption 2.4. All the initial curves are pairwise non-intersecting and locally self intersection free in the local sense, namely, there exist $\epsilon>0$ such that if

$$
C\left(t_{1}\right)=C\left(t_{2}\right), \quad\left|t_{1}-t_{2}\right|<\epsilon,
$$

then $t_{1}=t_{2}$.

We are now ready to introduce our family of subdivision schemes refining curves.

\section{The Smooth Facet Cutting Scheme}

\subsection{The Refinement Step}

The facet cutting scheme refines a given finite sequence of curves $\left\{C_{i}\right\}_{i=0}^{n}$ satisfying all the assumptions of the above with correspondences $\left\{v_{i}\right\}_{i=0}^{n-1}$ between consecutive pairs of curves to generate the finite sequence of sets $\left\{\bar{C}_{i}\right\}_{i=0}^{2 n-1}$ according to

$$
\begin{gathered}
\bar{C}_{2 i}(t)=(1-\mu) C_{i}(t)+\mu C_{i+1}\left(v_{i}(t)\right), \quad t \in I\left(C_{i}\right) ; \\
\bar{C}_{2 i+1}(t)=\mu C_{i}(t)+(1-\mu) C_{i+1}\left(v_{i}(t)\right), \quad t \in I\left(C_{i}\right),
\end{gathered}
$$

for fixed $0<\mu<\frac{1}{3}$ and $i=0,1, \ldots, n-1$.

In the next section we introduce conditions on the curves $\left\{C_{i}\right\}_{i=0}^{n}$ and the correspondences $\left\{v_{i}\right\}_{i=0}^{n-1}$ guaranteeing that the sets $\left\{\bar{C}_{i}\right\}_{i=0}^{2 n-1}$ in (3.1) are curves.

For the sake of simplicity, we assume that the refinement parameter $\mu$ is fixed. Yet, all the results are valid for varying $\mu$ bounded away from 0 and $\frac{1}{3}$.

Let $\mathcal{S}_{\mu}$ denote the refinement step operator generating $\left\{\bar{C}_{i}\right\}_{i=0}^{2 n-1}$ from $\left\{C_{i}\right\}_{i=0}^{n}$,

$$
\left\{\bar{C}_{i}\right\}_{i=0}^{2 n-1}=\mathcal{S}_{\mu}\left\{C_{i}\right\}_{i=0}^{n} .
$$

The repeated application of the refinement operator $\mathcal{S}_{\mu}$ is called The facet cutting scheme. The curves generated by the $j$-th step of the refinement are denoted by $\left\{C_{i}^{j}\right\}_{i=0}^{n_{j}}$. Repeating the refinement step in the fashion of (3.2) $j$ times one gets,

$$
\left\{C_{i}^{j}\right\}_{i=0}^{n_{j}}=\mathcal{S}_{\mu}\left\{C_{i}^{j-1}\right\}_{i=0}^{n_{j-1}}=\ldots=\mathcal{S}_{\mu}^{j}\left\{C_{i}^{0}\right\}_{i=0}^{n_{0}}
$$

where $\mathcal{S}_{\mu}^{j}$ is a shorthand notation for the operation of the refinement operator $j$ times.

In the remainder of the paper we will introduce conditions that will enable us to repeatedly apply the refinement operator $\mathcal{S}_{\mu}$ on the refined curves, and show the convergence of the scheme to a surface. Furthermore, we will show that under additional conditions, the scheme converges to a smooth surface.

\subsection{The Inherited Correspondence}

An essential tool for the analysis of the scheme is the inherited correspondence.

Definition 3.1. The correspondences vector for $\left\{\bar{C}_{i}\right\}_{i=0}^{2 n-1}$ in (3.1) based on their parametrization introduced there with $t \in I\left(C_{i}\right)$,

$$
v_{2 i}^{h}(t)=t, \quad i=0, \ldots, n-1, \quad v_{2 i+1}^{h}(t)=v_{i}(t), \quad i=0, \ldots, n-2,
$$

is called the inherited correspondence vector $\mathbf{v}^{h}=\left\{v_{i}^{h}\right\}_{i=0}^{2 n-2}$. For the inverse of $v_{i}^{h}$ we use the notation $v_{i}^{[h,-1]}$.

The inherited correspondence $v_{2 i}^{h}$ determines the pairing $\left(\bar{C}_{2 i}(t), \bar{C}_{2 i+1}(t)\right)$ while $v_{2 i+1}^{h}$ determines the paring $\left(\bar{C}_{2 i}(t), \bar{C}_{2 i+2}\left(v_{i}(t)\right)\right)$.

In fact, our condition on the correspondences at each refinement level, guaranteeing convergence and later smoothness, are requiring the correspondences not to be too "far" from the inherited ones.

We note that the inherited correspondence does not reduce to an arc length parametrization even when all the initial curves have the same length (we do not assume that), as is demonstrated in Example 3.2. 
Example 3.2. Consider the arc length curves

$$
C_{1}=(t, 0,0), \quad C_{2}=\left(\frac{t}{\sqrt{2}}, \frac{t}{\sqrt{2}}, 1\right), \quad I\left(C_{1}\right)=I\left(C_{2}\right)=[0,1]
$$

with the correspondence $v(t)=t$ (this is clearly a smooth correspondence). The generated curves are

$$
\begin{aligned}
\bar{C}_{1}(t) & =\left((1-\mu) t+\mu \frac{t}{\sqrt{2}}, \mu \frac{t}{\sqrt{2}}, \mu\right), \\
\bar{C}_{2}(t) & =\left(\mu t+(1-\mu) \frac{t}{\sqrt{2}},(1-\mu) \frac{t}{\sqrt{2}},(1-\mu)\right) .
\end{aligned}
$$

It is straightforward that $C_{1}$ and $C_{2}$ are curves for $0 \leq 0 \leq 1$. Yet, the parametrization $t$ is clearly not the arc length parametrization of them.

\section{The Piecewise Ruled Surface}

An important structure for our analysis is the piecewise ruled surface, which is analogous to the polyline in the case of point refining schemes.

Definition 4.1. Whenever the set

$$
R(C, \widetilde{C} ; v)=\{\varrho C(t)+(1-\varrho) \widetilde{C}(v(t)), \quad 0 \leq \varrho \leq 1, \quad t \in I(C)\}
$$

is a surface, it is called the ruled surface of the pair of parametrized curves $(C, \widetilde{C})$ under the correspondence $v$. For a parametrized curves vector $\left\{C_{i}\right\}_{i=0}^{n}$ and a correspondences vector $\mathbf{v}=\left\{v_{i}\right\}_{i=0}^{n-1}$ between consecutive curves, we define,

$$
P R=P R\left(\left\{C_{i}\right\}_{i}^{n} ; \mathbf{v}\right)=\bigcup_{i=0}^{n-1} R\left(C_{i}, C_{i+1} ; v_{i}\right) .
$$

$P R$ is called a piecewise ruled surface whenever each of its components is a surface.

We impose condition on $\left\{C_{i}\right\}_{i=0}^{n}$ and $\mathbf{v}$ guaranteeing that $P R$ is indeed a surface. For that we introduce the notion of cones. For a set of vectors $\left\{w_{i}\right\}_{i=1}^{m}$ in $\mathbb{R}^{3}$ let

$$
C o\left(\left\{w_{i}\right\}_{i=1}^{m}\right)=\left\{\sum_{i=1}^{m} a_{i} w_{i}, \quad a_{i} \in \mathbb{R}_{+}\right\},
$$

denote the cone generated by the vectors $\left\{w_{i}\right\}_{i=1}^{m}$.

One can easily verify that $C o\left(\left\{w_{i}\right\}_{i=1}^{m}\right)$ is the minimal cone which contains $\left\{w_{i}\right\}_{i=1}^{m}$.

In order to ensure that $R(C, \widetilde{C}, v)$ is indeed a surface we need the following definition:

Definition 4.2. Two cones $C o_{1}$ and $C o_{2}$ in $\mathbb{R}^{3}$ that satisfy for any $w_{1}, w_{2} \in C o_{1} \backslash\{\overrightarrow{0}\}$ and $u_{1}, u_{2} \in C o_{2} \backslash\{\overrightarrow{0}\}$,

$$
\frac{\left\langle w_{1} \times u_{1}, w_{2} \times u_{2}\right\rangle}{\left\|w_{1}\right\|\left\|u_{1}\right\|\left\|w_{2}\right\|\left\|u_{2}\right\|}>\beta
$$

with a constant $\beta>0$, are called $\beta$-transversal cones.

Definition 4.3. Let $\left\{C_{i}\right\}_{i=0}^{n}$ be curve vector with a correspondence vector $\mathbf{v}$. Then

$$
\Xi_{i}^{[\mathbf{v},+]}(t)=\frac{C_{i+1}\left(v_{i}(t)\right)-C_{i}(t)}{\left\|C_{i+1}\left(v_{i}(t)\right)-C_{i}(t)\right\|}, \quad \Xi_{i}^{[\mathbf{v},-]}(t)=\frac{C_{i}(t)-C_{i-1}\left(v_{i-1}^{-1}(t)\right)}{\left\|C_{i}(t)-C_{i-1}\left(v_{i-1}^{-1}(t)\right)\right\|}
$$

are called the forward secant and the backward secant of $C_{i}$ with respect to $\mathbf{v}$ at $t$.

One can easily see that

$$
\Xi_{i}^{[\mathbf{v},+]}(t)=\Xi_{i+1}^{[\mathbf{v},-]}\left(v_{i}(t)\right), \quad t \in I\left(C_{i}\right), \quad i=0, \ldots, n-1 .
$$

Remark 4.4. By Assumption 2.4 we have that $\Xi_{i}^{[\mathbf{v},+]}(t)$ and $\Xi_{i}^{[\mathbf{v},-]}(t)$ are well defined points on the unit sphere $\left(\mathbb{S}^{2}\right)$.

To measure the "quality" of a correspondence we need the next definition. 
Definition 4.5. Let $C(t)$ and $\widetilde{C}(\widetilde{t})$ be two curves with arc length parametrization $t$ and $\widetilde{t}$ respectively, and let $v$ be a correspondence between the curves $C$ and $\widetilde{C}$. Then

$$
\angle(\dot{C}(t), \dot{\widetilde{C}}(v(t)))=\arccos (\langle\dot{C}(t), \dot{\widetilde{C}}(v(t))\rangle), \quad t \in I(C),
$$

is the angle between $\dot{\mathbf{C}}(\mathbf{t})$ and $\dot{\widetilde{\mathbf{C}}}(\widetilde{\mathbf{t}})$ at $\mathbf{t}$ with respect to the correspondence $\mathbf{v}$, with the angle taken in the interval $[0, \pi)$. The $\beta$-transversal condition implies that there are no negative angles.

We note that the angle between two vectors on the sphere is equivalent to their geodesic distance.

In the previous attempt to design facet cutting scheme [13] the notion of orientable curves with respect to a correspondence was introduce. This notion ensures that (4.1) is a surface. This concept does not use the differential structure of the correspondence, and therefore it is intricate to work with. To overcome this difficulty we introduce next a similar notion which is easier to work with.

Definition 4.6. Let $\left\{C_{i}\right\}_{i=0}^{n}$ be a curves vector and let $\mathbf{v}$ be a sequence of correspondences. Iffor some $\beta>0$ the cones

$$
\mathfrak{C o}\left(\Xi_{i}^{[\mathbf{v},-]}(t), \Xi_{i}^{[\mathbf{v},+]}(t)\right), \quad \mathfrak{C o}\left(\dot{C}_{i-1}\left(v_{i-1}^{-1}(t)\right), \dot{C}_{i}(t), \dot{C}_{i+1}\left(v_{i}(t)\right)\right)
$$

are $\beta$-transversal cones for every $i$ and $t$, then $\mathbf{v}$ is called a regular correspondence.

Example 4.7. For the curves

$$
\begin{gathered}
C_{1}=(\sin (t), \cos (t), 0), \quad I\left(C_{1}\right)=[0, \pi] ; \\
C_{2}=(\sin (2 t), \cos (2 t), 1), \quad I\left(C_{2}\right)=\left[0, \frac{\pi}{2}\right],
\end{gathered}
$$

the correspondence $v_{1}(t)=\frac{1}{2} t$ is a regular correspondence. However, the correspondence $v_{2}(t)=\frac{\pi}{2}-\frac{1}{2} t$ is not regular.

The piecewise ruled surface is a well defined surface if it is intersection free in a local sense, i.e., if for $0 \leq \varrho_{1}, \varrho_{2} \leq 1$ and $\left|t_{1}-t_{2}\right|<\epsilon$ for some positive $\epsilon$ the equality

$$
\varrho_{1} C_{i}\left(t_{1}\right)+\left(1-\varrho_{1}\right) C_{i+1}\left(v_{i}\left(t_{1}\right)\right)=\varrho_{2} C_{i}\left(t_{2}\right)+\left(1-\varrho_{2}\right) C_{i+1}\left(v_{i}\left(t_{2}\right)\right),
$$

implies $\varrho_{1}=\varrho_{2}$ and $t_{1}=t_{2}$. We term this property as the local intersection free property (LIF property).

The forward or backward secant and the tangent at each point on a curve define a local coordinate system whenever they are linearly independent. The next lemma is needed for that fact.

Lemma 4.8. Let $C$ and $\widetilde{C}$ be two curves with a regular correspondences $v$. Then $R(C, \widetilde{C} ; v)$ is a surface.

The proof is a direct conclusion of Cohen et al. [6] Lemma 2.

Lemma 4.9. Let $\left\{C_{i}\right\}_{i=0}^{n}$ be a curves vector with a regular correspondences vector $\mathbf{v}$. Then $P R\left(\left\{C_{i}\right\} ; \mathbf{v}\right)$ as defined in (4.2) is a surface.

Proof. Since the curves and the correspondences are continuous it is enough to show that each $R\left(C_{i}, C_{i+1} ; v_{i}\right)$, for each $i=0 \ldots n-1$ has the LIF property. We note that the curves $C_{1}$ and $C_{2}$ of example 4.7 with the correspondence $v_{1}$ satisfy the LIF property. The $\beta$-transversal condition implies that

$$
\left\langle C_{i}(t) \times \Xi_{i}^{[\mathbf{v},+]}(t), C_{i+1}\left(v_{i}(t)\right) \times \Xi_{i}^{[\mathbf{v},+]}(t)\right\rangle>0 .
$$

Thus, by 4.8 the ruled surface $R\left(C_{i}, C_{i+1} ; v_{i}\right)$ is self-intersection free in the local sense.

Corollary 4.10. The parametrization of $P R\left(\left\{C_{i}\right\}_{i=0}^{n} ; \mathbf{v}\right)$ induced by (4.1) is a well defined parametrization for any correspondences vector $\mathbf{v}$.

Note that whenever $P R\left(\left\{C_{i}\right\} ; \mathbf{v}\right)$ is a surface with the LIF property the sets $\left\{\bar{C}_{i}\right\}_{i=0}^{2 n-1}$ in $(3.1)$ generated by the refinement operator $\mathcal{S}_{\mu}$, are well defined curves in $\mathbb{R}^{3}$.

\section{The Refined Piecewise Ruled Surface}

In the previous section we proved that a single refinement step is a well defined operator under appropriate conditions on the curves and correspondence. Now we impose additional assumptions on the curves to ensure that the scheme is well defined namely that an infinite number of refinement steps can be performed.

The $\beta$-transversal condition in the definition of the regular correspondence ensures that $P R=P R\left(\left\{C_{i}^{j}\right\}_{i=0}^{n_{j}}, \mathbf{v}^{j}\right)$ is a surface, and the facet cutting refinement yields a set of refined curves.

To be able to refine the refined curves we need to ensure that the set (4.2) generated by the refined curves is a regular surface. The next lemma provides this. 
Lemma 5.1. Consider the piecewise ruled surface $P R\left(\left\{C_{i}\right\}_{i=1}^{n} ; \mathbf{v}\right)$ where the vector of correspondences $\mathbf{v}$ is regular. Then $P R\left(\left\{\bar{C}_{i}\right\}_{i=0}^{2 n-1} ; \mathbf{v}^{h}\right)$ is a surface.

For the proof we need two lemmas.

Lemma 5.2. Consider the corner cutting scheme for points (1.1). Note that at refinement level $j n_{j+1}=2 n_{j}-1$. Let

$$
\mathfrak{D}_{i}^{j}=p_{i+1}^{j}-p_{i}^{j}, \quad i=0, \ldots, n_{j}-1, \quad j \in \mathbb{Z}_{+} .
$$

Then

$$
\mathfrak{D}_{2 i}^{j+1}=(1-2 \mu) \mathfrak{D}_{i}^{j}, \quad \mathfrak{D}_{2 i+1}^{j+1}=\mu\left(\mathfrak{D}_{i}^{j}+\mathfrak{D}_{i+1}^{j}\right) .
$$

The next lemma investigates the angle in the generated control polygons.

Lemma 5.3. Let

$$
\theta_{j}=\max _{i=0, \ldots, n_{j}-1} \angle\left(\mathfrak{D}_{i}^{j}, \mathfrak{D}_{i+1}^{j}\right), \quad \mathfrak{r}_{j}=\max _{i=0, \ldots, n_{j}-1} \max \left\{\frac{\left\|\mathfrak{D}_{i}^{j}\right\|}{\left\|\mathfrak{D}_{i+1}^{j}\right\|}, \frac{\left\|\mathfrak{D}_{i+1}^{j}\right\|}{\left\|\mathfrak{D}_{i}^{j}\right\|}\right\}
$$

Then

$$
\mathfrak{r}_{j+1} \leq \max \left\{\frac{\mu}{1-2 \mu}\left(1+\mathfrak{r}_{j}\right), \mathfrak{r}_{j} \frac{(1-2 \mu)}{\mu \sqrt{2\left(1+\cos \left(\theta_{j}\right)\right)}}\right\} .
$$

Proof of Lemma 5.1. If $C o_{1}$ and $C o_{2}$ are $\beta$-transversal cones then every sub-cone $\overline{C o}_{1}$ of $C o_{1}$ is $\beta$-transversal to $C o_{2}$. Indeed, by (3.1),

$$
\mathfrak{C o}\left(\dot{\bar{C}}_{2 i-1}\left(v_{i-1}^{-1}(t)\right), \dot{\bar{C}}_{2 i}(t), \dot{\bar{C}}_{2 i+1}(t)\right) \subset \mathfrak{C o}\left(\dot{C}_{i-1}\left(v_{i-1}^{-1}(t)\right), \dot{C}_{i}(t), \dot{C}_{i+1}\left(v_{i}(t)\right)\right)
$$

Consider the secants of the sequence of curves $\left\{\bar{C}_{i}\right\}_{i=0}^{2 n-1}$ with respect to $\mathbf{v}^{h}$,

$$
\bar{\Xi}_{2 i}^{\left[\mathbf{v}^{h},+\right]}(t)=\frac{\bar{C}_{2 i+1}(t)-\bar{C}_{2 i}(t)}{\left\|\bar{C}_{2 i+1}(t)-\bar{C}_{2 i}(t)\right\|}, \quad \bar{\Xi}_{2 i}^{\left[\mathbf{v}^{h},-\right]}(t)=\frac{\bar{C}_{2 i}(t)-\bar{C}_{2 i-1}\left(v_{i-1}^{[h,-1]}(t)\right)}{\left\|\bar{C}_{2 i}(t)-\bar{C}_{2 i-1}\left(v_{i-1}^{[h,-1]}(t)\right)\right\|}
$$

Lemma 5.2 ensures that

$$
\mathfrak{C o}\left(\Xi_{2 i}^{\left[\mathbf{v}^{h},-\right]}(t), \Xi_{2 i}^{\left[\mathbf{v}^{h},+\right]}(t)\right) \subset \mathfrak{C o}\left(\Xi_{i}^{[\mathbf{v},-]}(t), \Xi_{i}^{[\mathbf{v},+]}(t)\right) .
$$

On the ground of that, the cones

$$
\mathfrak{C o}\left(\dot{\bar{C}}_{2 i-1}^{j}\left(v_{i-1}^{-1}(t)\right), \dot{C}_{2 i}(t), \dot{C}_{2 i+1}(t)\right), \quad \mathfrak{C o}\left(\Xi_{2 i}^{\left[\mathbf{v}^{h},-\right]}(t), \bar{\Xi}_{2 i}^{\left[\mathbf{v}^{h},+\right]}(t)\right)
$$

are $\beta$-transversal cones. Therefore, the correspondences vector $\mathbf{v}^{h}$ is regular. Hence, Lemma 4.9 ensures that $P R\left(\left\{\bar{C}_{i}\right\}_{i=0}^{2 n-1} ; \mathbf{v}^{h}\right)$ is a regular surface, and the curves $\left\{\bar{C}_{i}\right\}_{i=0}^{2 n-1}$ can be refine with $\mathbf{v}^{h}$.

\section{Properties of a Single Refinement Step}

In this section we derive condition on $\left\{C_{i}\right\}_{i=0}^{n}$ and $\mathbf{v}=\left\{v_{i}\right\}_{i=0}^{n-1}$ such that $P R\left(\left\{\bar{C}_{i}\right\}_{i=0}^{2 n-1} ; \mathbf{v}^{h}\right)$ is "smoother" than $\left.P R\left(\left\{C_{i}\right\}_{i=0}^{n}\right\} ; \mathbf{v}\right)$ in a certain sense. Some of the proofs are deferred to the Appendix.

Definition 6.1. Let $C(t)$ and $\tilde{C}(\widetilde{t})$ be two curves with parametrizations $t$ and $\tilde{t}$ respectively. Let $v$ be a regular correspondence that satisfies

$$
\max _{t} \angle(\dot{C}(t), \dot{\tilde{C}}(v(t))) \leq \zeta, \quad \frac{1}{M} \leq \frac{d}{d t} v(t) \leq M
$$

for some

$$
0 \leq \zeta<\frac{\pi}{4}, \quad 1 \leq M<\infty .
$$

Then $v$ is called $a(\mathbf{Z}, \mathbf{M})$ correspondence of $C$ and $\widetilde{C}$. A correspondences vector $\mathbf{v}$ of $\left\{C_{i}\right\}_{i=0}^{n}$ such that each $v_{i}$ is a $(\zeta, M)$ correspondence of $C_{i}$ and $C_{i+1}$ is named $a(\mathbf{Z}, \mathbf{M})$ correspondence vector for $\left\{C_{i}\right\}_{i=0}^{n}$. We denote by $\mathbf{V}\left(\left\{C_{i}\right\}_{i=0}^{n} ; \zeta, M\right)$ the class of all the $(\zeta, M)$ correspondence vectors of $\left\{C_{i}\right\}_{i=0}^{n}$.

We point out that if $\mathbf{v}$ is a $(\zeta, M)$ correspondence vector of the curves $\left\{C_{i}\right\}_{i=0}^{n}$, then the correspondence vector $\mathbf{v}^{-1}=$ $\left(v_{n-1}^{-1}, \ldots, v_{0}^{-1}\right)$ is a $(\zeta, M)$ correspondence vector of the sequence of curves $\left\{C_{i}\right\}_{i=n}^{0}$.

Assumption 6.2. For the curves $\left\{C_{i}\right\}_{i=0}^{n}$ there exist $\zeta<\frac{\pi}{4}$ and $1 \leq M<\infty$ such that the class $\mathbf{V}\left(\left\{C_{i}\right\}_{i=0}^{n} ; \zeta, M\right)$ is not empty. 
One can verify that the curves in the next example satisfy Assumption 6.2.

Example 6.3. Consider the three planer curves

$$
C_{1}(t)=\frac{1}{\sqrt{2}}(t, t, 0), \quad C_{2}(t)=\frac{1}{\sqrt{3.25}}(t, 1.5 t, 0) \quad C_{3}(t)=\frac{1}{\sqrt{5}}(t, 2 t, 0),
$$

with the identity correspondence $(M=1)$ and the angle $\zeta$ is 0.1974 radian.

Now we present two results guaranteeing that the refined curves $\left\{\bar{C}_{i}\right\}_{i=0}^{2 n-1}$ of (3.1) satisfy Assumption 6.2 if the initial curves $\left\{C_{i}\right\}_{i=0}^{n}$ satisfy this assumption with some constrains.

Theorem 6.4. Let $\left\{C_{i}\right\}_{i=0}^{n}$ be a sequence of curves satisfying Assumption 6.2 , let $\mathbf{v} \in \mathbf{V}\left(\left\{C_{i}\right\}_{i=0}^{n} ; \zeta, M\right)$ and let $\left\{\bar{C}_{i}\right\}_{i=0}^{2 n-1}$ be defined as in (3.1).

Assume that

$$
\zeta<\min \left\{\sqrt{2 \frac{1-\mu}{\mu}-2}, \sqrt{\frac{2 \mu}{1-\mu}}, \frac{\pi}{4}\right\} .
$$

If there exist $\lambda(\mu, \zeta)>0$ such that

$$
M \leq \min \left\{\frac{2 \mu}{(1-\mu)\left(\zeta^{2}+2 \lambda(\mu, \zeta)\right)}, \frac{2(1-\mu)}{\mu\left(2+4 \lambda(\mu, \zeta)+\zeta^{2}\right)}\right\},
$$

then

$$
\bar{\zeta}=\max _{i, t} \angle\left(\dot{\bar{C}}_{i}(t), \dot{\bar{C}}_{i+1}\left(v_{i}^{h}(t)\right)\right)<\frac{\zeta}{1+\lambda(\mu, \zeta)}<\frac{\pi}{4} .
$$

The proof of this theorem is given in Appendix A.

Note that (6.2) ensures that condition (6.3) does not contradict the requirement $M \geq 1$ in Assumption 6.2. To show that $V\left(\left\{\bar{C}_{i}\right\}_{i=0}^{2 n-1} ; \bar{\zeta}, \bar{M}\right)$ is not empty it remains to show in view of Theorem 6.4, that there exists a finite $\bar{M}$ defined as in (6.1) with respect to $\mathbf{v}^{h}$. This is done in Theorem 6.6. Both the theorem and the next lemma are proved in Appendix A.

Lemma 6.5. Let $\tau_{2 i}, \tau_{2 i+1}$ and $\tau_{2 i-1}$ be the arc length parametrizations of the curves $\bar{C}_{2 i}, \bar{C}_{2 i+1}$ and $\bar{C}_{2 i-1}$ respectively. Then for all possible $i$ and $t$ we have,

$$
\begin{gathered}
\sqrt{\cos (\zeta)}\left(1-\mu+\mu \frac{d}{d t} v_{i}(t)\right) \leq \frac{d}{d t} \tau_{2 i}(t) \leq 1-\mu+\mu \frac{d}{d t} v_{i}(t) \\
\sqrt{\cos (\zeta)}\left(\mu+(1-\mu) \frac{d}{d t} v_{i}(t)\right) \leq \frac{d}{d t} \tau_{2 i+1}(t) \leq \mu+(1-\mu) \frac{d}{d t} v_{i}(t)
\end{gathered}
$$

and

$$
\sqrt{\cos (\zeta)}\left(1-\mu+\mu \frac{d}{d t} v_{i-1}^{-1}(t)\right) \leq \frac{d}{d t} \tau_{2 i-1}(t) \leq 1-\mu+\mu \frac{d}{d t} v_{i-1}^{-1}(t) .
$$

Next we obtain bounds on the derivative of $v_{i}^{h}$ with respect to the arc length parametrization of $\bar{C}_{i}$, for $i=0,1 \ldots, 2 n-2$.

Theorem 6.6. Let $\left\{C_{i}\right\}_{i=0}^{n}$ satisfy Assumption 6.2, let $\tau_{i}$ denote the arc-length parametrization of the curve $\bar{C}_{i}$, $i=$ $0,1, \ldots, 2 n-1$, and let

$$
\bar{M}=\max _{i, \tau_{i}}\left\{\frac{d}{d \tau_{i}} v_{i}^{h}\left(\tau_{i}\right),\left(\frac{d}{d \tau_{i}} v_{i}^{h}\left(\tau_{i}\right)\right)^{-1}\right\} .
$$

Then $v^{h} \in V\left(\left\{\bar{C}_{i}\right\}_{i=0}^{2 n-1}, \bar{\zeta}, \bar{M}\right)$ with $\bar{\zeta}$ as in (6.4) and

$$
\bar{M} \leq \frac{1+\mu^{*}(M-1)}{\sqrt{\cos (\zeta)}}<\frac{M}{\sqrt{\cos (\zeta)}}, \quad \mu^{*}=\max \{2 \mu, 1-2 \mu\} .
$$

An important property of the refined curves is the Hölder continuity of their derivatives.

Lemma 6.7. For a sequence of curves $\left\{C_{i}\right\}_{i=0}^{n}$ and a correspondence vector $\mathbf{v} \in \mathbf{V}\left(\left\{C_{i}\right\}_{i=0}^{n} ; \zeta, M\right)$ let $\left\{\bar{C}_{i}\right\}_{i=0}^{2 n-1}$ be defined as in (3.1). The following inequality holds

$$
\max _{i} \mathrm{H}_{t a n}\left(\bar{C}_{i}\right) \leq \max _{i} \mathrm{H}_{t a n}\left(C_{i}\right)
$$

The proof is omitted since it is a trivial use of the triangle inequality and the fact that the correspondence is smooth. It follows from Theorems 6.4 and 6.6 that the refined curves satisfy Assumption 6.2 and thus can be refined.

Lemma 6.8. Using the notation introduced in Theorem 6.4 , consider two correspondence vectors $\mathbf{v}^{[1]}, \mathbf{v}^{[2]} \in \mathbf{V}\left(\left\{C_{i}\right\}_{i=0}^{n} ; \zeta, M\right)$. Then for all $i=0,1, \ldots, n-1$,

$$
\max _{t}\left\|C_{i+1}\left(v_{i}^{[1]}(t)\right)-C_{i+1}\left(v_{i}^{[2]}(t)\right)\right\| \leq \mathrm{L} \frac{M^{2}-1}{M}, \quad 0 \leq t \leq \operatorname{length}\left(C_{i}\right)
$$

where $\mathrm{L}=\max _{i}$ length $\left(C_{i}\right)$. 
Proof. First we investigate the quantity $\max _{t}\left|v_{i}^{[1]}(t)-v_{i}^{[2]}(t)\right|$, where $t$ is the arc length parametrization of $C_{i}$. As shown in our previous work [13] (joint work with Dyn) we have,

$$
\operatorname{length}\left(\bar{C}_{i}\right) \leq \mathrm{L}<\infty, \quad i=0, \ldots, 2 n-1 .
$$

Since the parametric interval of arc length parametrization is $\left[0, I\left(C_{i}\right)\right]$ one can see that $v_{i}^{[1]}(0)=v_{i}^{[2]}(0)=0$.

By the assumptions on $\mathbf{v}^{[1]}$ and $\mathbf{v}^{[2]}$, and by the fundamental theorem of calculus we get

$$
\frac{t}{M} \leq v_{i}^{[1]}(t), v_{i}^{[2]}(t) \leq M t, \quad t \in I\left(C_{i}\right) .
$$

Thus,

By basic vector calculus

$$
\max _{t \in I\left(C_{i}\right)}\left|v_{i}^{[1]}(t)-v_{i}^{[2]}(t)\right| \leq \mathrm{L}\left(M-\frac{1}{M}\right), \quad i=0,1, \ldots, n-1
$$

$$
\begin{gathered}
\left\|C_{i+1}\left(v_{i}^{[1]}(t)\right)-C_{i+1}\left(v_{i}^{[2]}(t)\right)\right\|=\left\|\int_{v_{i}^{[1]}(t)}^{v_{i}^{[2]}(t)} \dot{C}_{i+1}(\tau) d \tau\right\| \\
\leq \int_{v_{i}^{[1]}(t)}^{v_{i}^{[2]}(t)} d \tau\left|v_{i}^{[1]}(t)-v_{i}^{[2]}(t)\right| \leq \frac{M^{2}-1}{M} \mathrm{~L} .
\end{gathered}
$$

\section{The Repeated Refinement Step}

Now we are ready to study the repeated refinement steps. Let us first update our notation. Recall that we denote by $\left\{C_{i}^{j}\right\}_{i=0}^{n_{j}}$ the curves generated by the $j$-th refinement step of our scheme as defined in (3.3). Furthermore, we denote by $\mathbf{v}^{j}=\left\{v_{i}^{j}\right\}_{i=0}^{n_{j}-1}$ the correspondence vector at the $j+1^{\text {st }}$ refinement step.

We use the shorthand notation $P R^{j}$ for $P R\left(\left\{C_{i}^{j}\right\}_{i=0}^{n_{j}} ; \mathbf{v}^{j}\right)$. Moreover, for the secants we use the notation

$$
\Xi_{i}^{[j,+]}(t)=\frac{C_{i+1}^{j}\left(v_{i}^{j}(t)\right)-C_{i}^{j}(t)}{\left\|C_{i+1}^{j}\left(v_{i}^{j}(t)\right)-C_{i}^{j}(t)\right\|}, \quad \Xi_{i}^{[j,-]}(t)=\frac{C_{i}^{j}(t)-C_{i-1}^{j}\left(v_{i-1}^{[j,-1]}(t)\right)}{\left\|C_{i}^{j}(t)-C_{i-1}^{j}\left(v_{i-1}^{[j,-1]}(t)\right)\right\|} .
$$

In addition we introduce the three quantities,

$$
\begin{aligned}
& \zeta_{j}=\max _{i, t} \angle\left(\dot{C}_{i}^{j}(t), \dot{C}_{i+1}^{j}\left(v_{i}^{j}(t)\right)\right) ; \\
& M_{j}=\max _{i, t}\left\{\frac{d}{d t} v_{i}^{j}(t),\left(\frac{d}{d t} v_{i}^{j}(t)\right)^{-1}\right\} ; \\
& \xi_{j}=\max _{i, t} \angle\left(\Xi_{i}^{\left[\mathbf{v}^{j},+\right]}(t), \Xi_{i}^{\left[\mathbf{v}^{j},-\right]}(t)\right) .
\end{aligned}
$$

Since the curves are contained in a compact set and the correspondences are smooth all the maxima above are achieved.

The following definition is central to the analysis of convergence.

Definition 7.1. Given the sequence $(\mathbf{Z}, \mathbf{M})=\left\{\left(\zeta_{j}, M_{j}\right)\right\}_{j=0}^{\infty}$, with $0 \leq \zeta_{j}<\frac{\pi}{4}$ and $M_{j}>1$, let $\left\{C_{i}^{0}\right\}_{i=0}^{n_{0}}$ satisfy the conditions of Theorem 6.4 and let $\mathbf{v}^{j} \in \mathbf{V}\left(\left\{C_{i}^{j}\right\}_{i=0}^{n_{j}} ; \zeta_{j}, M_{j}\right)$. If

$$
\zeta_{j} \leq \frac{\zeta_{j-1}}{\left(1+\lambda\left(\mu, \zeta_{0}\right)\right)}, \quad M_{j} \leq \frac{\left.1+\mu^{*}\left(M_{j-1}-1\right)\right\}}{\sqrt{\cos \left(\left(1+\lambda\left(\mu, \zeta_{0}\right)\right)^{-1} \zeta_{j-1}\right)}}
$$

then the sequence of correspondence vectors $\left\{\mathbf{v}^{j}\right\}_{j=0}^{\infty}$ is called a $(\mathbf{Z}, \mathbf{M})$ admissible sequence of correspondence vectors for $\left\{C_{i}^{0}\right\}_{i=0}^{n_{0}}$.

In the following we will show that the class of $(\mathbf{Z}, \mathbf{M})$ admissible sequence of correspondence vectors is not empty, and that by choosing a correspondence sequence in this class we ensure the convergence of the sequences $\left\{\dot{C}_{i}^{j}(t)\right\}_{j=0}^{\infty}$ and therefore of the scheme.

From this point we are using the shorthand notation $\lambda$ for $\lambda\left(\mu, \zeta_{0}\right)$.

To grantee that $M_{j}$ for $j \geq 0$ satisfy (6.3) with $\zeta=\zeta_{0}$ we require a slightly stronger assumption on $M_{0}$.

Assumption 7.2. There exist $\lambda=\lambda\left(\mu, \zeta_{0}\right)>0$ such that for $\left\{C_{i}^{0}\right\}_{i=0}^{n_{0}}$

$$
\begin{gathered}
\exp \left(\frac{\zeta_{0}}{1-(1+\lambda)^{-1}}\right) M_{0} \\
<\min \left\{\frac{1-\mu}{\mu\left(1-\cos \left(\zeta_{0}\right)+\lambda\right)}, \frac{\mu}{(1-\mu)\left(2-\cos \left(\zeta_{0}\right)+2 \lambda\right)}\right\} .
\end{gathered}
$$


Theorem 7.3. Let the initial curves satisfy Assumptions 6.2 and 7.2, and assume further that there exists a sequence of admissible correspondence vectors $\mathbf{v}^{0}, \mathbf{v}^{1}, \ldots, \mathbf{v}^{k}$ such that

$$
\zeta_{j} \leq \frac{\zeta_{j-1}}{(1+\lambda)}, \quad M_{j} \leq \frac{1+\mu^{*}\left(M_{j-1}-1\right)}{\sqrt{\cos \left((1+\lambda)^{-1} \zeta_{j-1}\right)}}
$$

for $j=1,2, \ldots k$. Then there exists a correspondence vector $\mathbf{v}^{k+1} \in \mathbf{V}\left(\left\{C_{i}^{k+1}\right\}_{i=0}^{n_{k+1}}, M_{k+1}\right)$ with

$$
\zeta_{k+1} \leq \frac{\zeta_{k}}{(1+\lambda)}, \quad M_{k+1} \leq \frac{1+\mu^{*}\left(M_{k}-1\right)}{\sqrt{\cos \left((1+\lambda)^{-1} \zeta_{k}\right)}} .
$$

Proof. First we show that the theorem is true for $k=0$. By choosing the inherited correspondence Theorem 6.6 and Lemma ?? ensure that the conditions of Theorem 6.4 hold for $k=0$, which yields the existence of a correspondence vector $\mathbf{v}^{1}$ for which $\zeta_{1} \leq \frac{\zeta_{0}}{1+\lambda}$. Moreover, Theorem 6.6 implies that one can choose a correspondence that agrees with the above and for which

$$
M_{1} \leq \frac{1+\mu^{*}\left(M_{0}-1\right)}{\sqrt{\cos \left(\zeta_{0}\right)}} .
$$

Consequently, the class of $\left(\zeta_{1}, M_{1}\right)$ admissible correspondences after a single refinement step is not empty with $\zeta_{1} \leq \frac{\zeta_{0}}{1+\lambda}$ and $M_{1}$ satisfying (7.2). By repeated used of Theorems 6.4 and 6.6 by the use of basic calculus we get that

$$
M_{k+1} \leq M_{0} \prod_{l=0}^{j-1} \frac{1}{\sqrt{\cos \left(\zeta_{l}\right)}}<\exp \left(\frac{\zeta_{0}}{1-(1+\lambda)^{-1}}\right) M_{0} .
$$

Thus, $M_{j}$ is bounded for all $j>0$. By Assumption 7.2 the conditions of Theorem 6.4 hold. Therefore,

$$
\zeta_{k+1}<(1+\lambda)^{-1} \zeta_{k}
$$

for $\mathbf{v}^{k+1}=\mathbf{v}^{[k, h]}$. Similarly by Theorem 6.6 we get

$$
M_{k+1} \leq \frac{\left.1+\mu^{*}\left(M_{k}-1\right)\right)}{\sqrt{\cos \left((1+\lambda)^{-1} \zeta_{k}\right)}}
$$

proving that after the $k+1^{\text {st }}$ refinement step the class $V\left(\left\{C_{i}^{k+1}\right\}_{i=0}^{n_{k+1}} ; \zeta_{k+1}, M_{k+1}\right)$ is not empty, with the particular choice of $\zeta_{k+1}$ and $M_{k+1}$ of the above.

It is worth mentioning that we showed that the class of $\left(\mathbf{Z}_{k+1}, \mathbf{M}_{k+1}\right)$ admissible correspondences is not empty by choosing the inherited correspondence. But since this claim is closed under small perturbation this is a continuum number of correspondence vectors in this class.

\section{Convergence of the Scheme}

With the results of the previous subsection we can prove the convergence of the facet cutting scheme. This is done in the Hausdorff metric for sets. Nevertheless, we show that the correspondence sequence converges in a stronger sense than that.

The Hausdorff metric of compact sets $A$ and $B$, in $\mathbb{R}^{3}$ with respect to the Euclidean metric $d(\cdot, \cdot)$ of points in $\mathbb{R}^{3}$ is defined as,

$$
\operatorname{haus}(A, B)=\max \left\{\max _{a \in A} \min _{b \in B} d(a, b), \max _{b \in B} \min _{a \in A} d(a, b)\right\} .
$$

For a survey on the Hausdorff metric see Sendov [16].

We proceed with Lemmas which are essential for the analysis. Their proofs are in Appendix B.

Lemma 8.1. Under the condition of Theorems 6.4,6.6 and Lemma 6.5. Let $\left\{\mathrm{M}_{j}\right\}_{j=0}^{\infty}$ be the following recursive sequence,

$$
\mathrm{M}_{j+1}=\frac{\max \left\{f_{1}\left(\mathrm{M}_{j}\right), f_{2}\left(\mathrm{M}_{j}\right)\right\}}{\sqrt{\cos \left((1+\lambda(\mu, \zeta))^{-j} \zeta\right)}}, \quad \mathrm{M}_{0}>1, \quad 0 \leq \zeta<\frac{\pi}{4},
$$

where $\lambda(\mu, \zeta)>0$ is defined in Theorem 6.4 and the function $f_{1}$ and $f_{2}$ as defined as

$$
f_{1}(x)=\frac{(1-\mu) x+\mu}{\mu x+(1-\mu)}, \quad f_{2}(x)=x \frac{\mu x+(1-\mu)}{(1-\mu) x+\mu}, \quad x \in[1, \infty) .
$$

Then

$$
\sum_{j=0}^{\infty}\left(\mathrm{M}_{j}-1\right)<\infty
$$


By using a well known theorem in calculus [1] one can show that

$$
\prod_{j=0}^{\infty} \mathrm{M}_{j}<\infty \Longleftrightarrow \sum_{j=0}^{\infty}\left(\mathrm{M}_{j}-1\right)
$$

The next lemma plays an important role in the convergence analysis.

Lemma 8.2. Let $0 \leq \zeta_{0}<\frac{\pi}{4}$. Then for all $k \in \mathbb{Z}_{+}$

$$
\prod_{j=0}^{k} \sqrt{\cos \left(\frac{\zeta_{0}}{\left(1+\lambda\left(\mu, \zeta_{0}\right)\right)^{j}}\right)} \geq \exp \left(\frac{-\zeta_{0}}{1-\left(1+\lambda\left(\mu,, \zeta_{0}\right)\right)^{-1}}\right) .
$$

Theorem 8.3. The scheme with the sequence of $(\mathbf{Z}, \mathbf{M})$-admissible correspondences converges, i.e., the sequence $\left\{P R^{j}\right\}_{j=0}^{\infty}$ is a Cauchy sequence with respect to the Hausdorff metric.

Proof. Let

$$
d_{j}^{h}=\max _{i, t}\left\|C_{i}^{j}(t)-C_{i+1}^{j}\left(v_{i}^{[j, h]}(t)\right)\right\|, \quad d_{j}=\max _{i, t}\left\|C_{i}^{j}(t)-C_{i+1}^{j}\left(v_{i}^{j}(t)\right)\right\| .
$$

Using (3.3) and the definition of the ruled surfaces (Definition 4.1) we get

$$
\operatorname{haus}\left(P R^{j}, P R^{j+1}\right) \leq \mu d_{j}, \quad j \in \mathbb{Z}_{+} .
$$

Thus, to prove that the sequence $\left\{P R^{j}\right\}_{j=0}^{\infty}$ is a Cauchy sequence with respect to the Hausdorff metric it suffices to show that the sequence $\left\{d_{j}\right\}_{j=0}^{\infty}$ is summable, i.e., $\sum_{j=0}^{\infty} d_{j}<\infty$. As proved by the authors of the previous paper [13] (joint work with Dyn),

$$
d_{j}^{h} \leq \rho d_{j-1}, \quad j \in \mathbb{Z}_{+},
$$

with $\rho=\max \left\{\mu^{*}(1+\lambda)^{-2}\right\}$. By Lemma 6.8 , for any other $\left(\zeta_{j}, M_{j}\right)$ admissible correspondence sequence we have

$$
d_{j} \leq \rho d_{j-1}+\left(M_{j}^{2}-1\right) \mathrm{L} .
$$

Consequently,

$$
d_{j} \leq \rho^{j} d_{0}+\mathrm{L} \sum_{l=0}^{j} \rho^{j-l}\left(M_{l}^{2}-1\right)
$$

According to the proof of Lemma 8.2

$$
d_{j} \leq \rho^{j-1}\left(\rho d_{0}+\mathrm{L} \sum_{l=0}^{j}\left(\left(M_{0}-1\right)\left(1+\frac{\zeta_{0}}{2}\right)+(l-1) \frac{\zeta_{0}}{2}\right)\right)
$$

which yields

$$
\sum_{j=0}^{\infty} d_{j} \leq \sum_{j=0}^{\infty} \rho^{j-1} N_{j}<\infty
$$

where

$$
N_{j}=\rho d_{0}+\mathrm{L}\left(j\left(M_{0}-1\right)\left(1+\frac{\zeta_{0}}{2}\right)+\frac{\zeta_{0} j(j-1)}{4}\right) .
$$

Thus, $\left\{d_{j}\right\}_{j=0}^{\infty}$ is summable.

We denote the limit set by $P R^{\infty}$.

In the next section we prove that $P R^{\infty}$ is indeed a surface.

\section{Parametrization of the Limit Surface} tion.

We prove that $P R^{\infty}$ is a surface by showing that it has a local parametrization. In this section we study this parametriza-

Definition 9.1. For $p \in C_{i}^{j}$, with the notation of Section 7, the offspring operator $T$ associates with $p$ its two direct offsprings

$$
T(p, k)=\left\{\begin{array}{cc}
C_{2 i}^{j+1}(t), & k=1 \\
C_{2 i-1}^{j+1}\left(v_{i-1}^{[j,-1]}(t)\right), & k=0
\end{array} .\right.
$$

with $k \in\{0,1\}$ and $a_{0} \in C_{i}^{0}$ where $t$ is the parameter in $I\left(C_{i}^{j}\right)$ such that $p=C_{i}^{j}(t)$. 
In (9.1) we use the parametrization of (3.1).

For every $0 \leq x<1$ we have the binary representation

$$
x=\sum_{j=1}^{\infty} x(j) 2^{-j}, \quad x(j) \in\{0,1\} .
$$

Using this we have the following definition,

Definition 9.2. For given $a_{0} \in C_{i}^{0}$ for some $i \in\left\{0,1, \ldots, n_{0}\right\}$ and $0 \leq x<1$ the recursive sequence of points in $\mathbb{R}^{3}$

$$
a_{j+1}\left(a_{0}, x\right)=T\left(a_{j}\left(a_{0}, x\right), x(j+1)\right), \quad j \in \mathbb{Z}_{+}
$$

is called the offspring sequence of $a_{0}$ with respect to $x$. Here $T$ is the offspring operator.

Note that by definition, $a_{j}\left(a_{0}, x\right)$ is a point on one of the curves $\left\{C_{i}^{j}\right\}_{i=0}^{n_{j}}$ and that

$$
\left\{C_{i}^{j}\right\}_{i=0}^{n_{j}}=\bigcup_{i=0}^{n_{0}} \bigcup_{a_{0} \in C_{i}^{0}} \bigcup_{x \in[0,1)} a_{j}\left(a_{0}, x\right) .
$$

Theorem 9.3. Every $a_{0} \in C_{i}^{0}, i=0, \ldots, n_{0}$ and $0 \leq x<1$ define a unique offspring sequence that converges to a limit $a\left(a_{0}, x\right)$ uniformly in $a_{0}$ and $x$. Moreover,

$$
\bigcup_{i=0}^{n_{0}} \bigcup_{a_{0} \in C_{i}^{0}} \bigcup_{0 \leq x<1} a\left(a_{0}, x\right)=P R^{\infty} .
$$

Proof. By (8.6) and (8.7) we have that the sequence $\left\{d_{j}\right\}_{j=0}^{\infty}$ is summable. Thus, the first part of the theorem follows from the relation,

$$
\left\|a_{j}\left(a_{0}, x\right)-a_{j+1}\left(a_{0}, x\right)\right\| \leq \mu d_{j} .
$$

Using arguments similar to those introduced by the author in [13] (joint work with Dyn) one can verify that,

$$
\operatorname{haus}\left(\left\{C_{i}^{j}\right\}_{i=0}^{n_{j}}, P R^{\infty}\right) \leq \sum_{l=j}^{\infty} d_{l} .
$$

Combining this with (9.3), the first part of the theorem and the summability of $\left\{d_{j}\right\}_{j=0}^{\infty}$, the second part of the theorem, namely (9.4), follows.

By using Theorem 9.3 we will show in the following that the limit set $P R^{\infty}$ is a continuous surface.

Theorem 9.4. The pair $\left(a_{0}, x\right)$ is a continuous local coordinate system for $P R^{\infty}$.

Proof. Theorem 9.3 states that for every $p \in P R^{\infty}$ there exists an offspring sequence $\left\{a_{j}\left(a_{0}, x\right)\right\}_{j=0}^{\infty}$ that converges to $p$. Thus, $\left(a_{0}, x\right)$ is a parametrization of $P R^{\infty}$.

All of the sequences $\left\{a_{j}\left(a_{0}, x\right)\right\}_{j=0}^{\infty}$ converge uniformly to their limit. Hence, $\left(a_{0}, x\right)$ is a local coordinate of $P R^{\infty}$, with $x_{0} \in[0,1)$ and $a_{0} \in\left\{C_{i}^{0}\right\}_{i=0}^{n_{0}}$.

Since all the sequences $\left\{a_{j}\left(a_{0}, x\right)\right\}_{j=0}^{\infty}$ converge uniformly to their limits, $\left(a_{0}, x\right)$ is a local continuous coordinate system.

Although several offspring sequences can converge to the same limit, the LIF condition and Assumption 2.1 ensures us, the different sequences correspond to separated initial points.

Following the path of the offsprings sequences we define the tangent offspring sequence,

$$
\operatorname{Tan}_{j}\left(a_{0}, x\right)=\dot{C}_{i}^{j}(t), \quad a_{j}\left(a_{0}, x\right)=C_{i}^{j}(t) .
$$

A direct consequence of Theorem 9.4 and the $\left\{\zeta_{j}\right\}_{j=0}^{\infty}$ decay econometric is the next corollary.

Corollary 9.5. The all the sequences $\left\{\operatorname{Tan}_{j}\left(a_{0}, x\right)\right\}_{j=0}^{\infty}$ converge uniformly in $a_{0}$ and $x$.

The fact that the piecewise ruled and their limit are all manifold we consider them locally as functions. Since the limit of uniformly converge continuous function is continuous we get that $\frac{\partial}{\partial a_{0}} P R^{\infty}$ is continuous. Moreover, one can prove that the derivative of in the $a_{0}$ direction satisfy Hölder condition with the same constant as the curves $\left\{C_{i}^{0}\right\}_{i=0}^{n_{0}}$. 


\section{Two Properties of the Generated Curves}

In this section we state a few properties of the generated curves.

An important consequence of Theorem 7.3 is

Theorem 10.1. Let $\left\{\mathbf{v}^{j}\right\}_{j=0}^{\infty}$ be a $(\mathbf{Z}, \mathbf{M})$ admissible correspondence sequence. Then

$$
\lim _{j \rightarrow \infty} \max _{i}\left|\operatorname{length}\left(C_{i}^{j}\right)-\operatorname{length}\left(C_{i+1}^{j}\right)\right|=0
$$

Proof. Let $t_{i}^{j}$ and $t_{i+1}^{j}$ be the arc length parametrization of $C_{i}^{j}$ and $C_{i+1}^{j}$ respectively. Then by the definition of length and of $M_{j}$ one can show that

$$
\begin{aligned}
\operatorname{length}\left(C_{i+1}^{j}\right) & =\int_{0}^{\operatorname{length}\left(C_{i+1}^{j}\right)}\left\|\dot{C}_{i+1}^{j}\left(t_{i+1}^{j}\right)\right\| d t_{i+1}^{j} \\
& =\int_{0}^{\operatorname{length}\left(C_{i}^{j}\right)}\left\|\dot{C}_{i+1}^{j}\left(v_{i}^{j}\left(t_{i}^{j}\right)\right) \frac{d v_{i}^{j}}{d t_{i+1}^{j}}\left(t_{i}^{j}\right)\right\| d t_{i}^{j} \\
& \leq \int_{0}^{\operatorname{length}\left(C_{i}^{j}\right)}\left\|\dot{C}_{i}^{j}\left(v_{i}^{j}\left(t_{i}^{j}\right)\right)\right\| M_{j} d t_{i}^{j} \\
& =M_{j} \operatorname{length}\left(C_{i}^{j}\right) .
\end{aligned}
$$

Similarly we get length $\left(C_{i+1}^{j}\right) \geq \frac{\operatorname{length}\left(C_{i}^{j}\right)}{M_{j}}$. The claim of the theorem follows from these two inequalities together with Theorem 7.3.

It is clear from the proof above that the correspondence as $j \rightarrow \infty$ "tend" to be isometries.

A direct consequence of Lemma 6.7 is,

Theorem 10.2. The Hölder constants of the arc length derivative of all the curves generated by the scheme are bounded uniformly.

\section{A Proofs of Section 6}

Proof of Theorem 6.4. First we prove that there exist $\lambda(\mu, \zeta)>0$ such that

$$
\bar{\zeta} \leq \frac{\zeta}{1+\lambda(\mu, \zeta)}
$$

Denote

$$
\theta_{i}(t)=\angle\left(\dot{C}_{i}(t), \dot{C}_{i+1}\left(v_{i}(t)\right)\right), \quad t \in I\left(C_{i}\right)
$$

and

$$
\bar{\theta}_{2 i}(t)=\angle\left(\dot{\bar{C}}_{2 i}(t), \dot{\bar{C}}_{2 i+1}(t)\right)
$$

By (3.1) we have,

$$
\dot{\bar{C}}_{2 i}(t), \dot{\bar{C}}_{2 i+1}(t) \in \mathfrak{C o}\left(\dot{C}_{i}(t), \dot{C}_{i+1}\left(v_{i}(t)\right)\right) .
$$

The geometry of the unit sphere in $\mathbb{R}^{3}$ implies that

$$
\dot{\bar{C}}_{2 i}(t), \dot{\bar{C}}_{2 i+1}(t) \in \mathfrak{C o}\left(\dot{C}_{i}(t), \dot{C}_{i+1}\left(v_{i}(t)\right)\right) \cap \mathbb{S}^{2}
$$

This leads to $\bar{\theta}_{2 i}(t) \leq \theta_{i}(t) \leq \zeta<\frac{\pi}{4}$. Moreover since $\theta_{i}(t)$ agrees with (6.2),

$$
\bar{\theta}_{2 i}(t)=\angle\left(\dot{\bar{C}}_{2 i}(t), \dot{\bar{C}}_{2 i+1}(t)\right) \leq \angle\left(\dot{C}_{i}(t), \dot{\bar{C}}_{2 i+1}(t)\right)=\omega<\theta_{i}(t)
$$

Now,

$$
\dot{\bar{C}}_{2 i+1}(t)=\alpha \dot{C}_{i}(t)+\beta u
$$

where $\left(\dot{C}_{i}(t), u\right)$ is an orthogonal basis in the spanned by $\dot{C}_{i}(t), \dot{C}_{i+1}\left(v_{i}(t)\right)$, with

$$
\begin{gathered}
\alpha=\mathcal{K}\left(\mu+(1-\mu) \frac{d}{d t} v_{i}(t) \cos \left(\theta_{i}(t)\right)\right. \\
\beta=\mathcal{K}(1-\mu) \frac{d}{d t} v_{i}(t) \sin \left(\theta_{i}(t)\right),
\end{gathered}
$$


where $\mathcal{K}$ is a positive number ensuring that $\left\|\dot{\bar{C}}_{2 i+1}(t)\right\|=1$.

In view of (A.3) we have

$$
\omega=\arctan \left(\frac{(1-\mu) \frac{d}{d t} v_{i}(t) \sin \left(\theta_{i}(t)\right)}{\mu+(1-\mu) \frac{d}{d t} v_{i}(t) \cos \left(\theta_{i}(t)\right)}\right) .
$$

By Taylor expansion,

$$
\arctan (\theta)<\theta, \quad 0<\theta<\frac{\pi}{4}
$$

which yields, since $0<\omega<\frac{\pi}{4}$,

$$
\omega \leq \frac{(1-\mu) \sin \left(\theta_{i}(t)\right) \frac{d}{d t} v_{i}(t)}{\mu+(1-\mu) \frac{d}{d t} v_{i}(t) \cos \left(\theta_{i}(t)\right)}
$$

Thus,

$$
\bar{\theta}_{2 i}(t)<\omega<\frac{(1-\mu) \sin \left(\theta_{i}(t)\right) \frac{d}{d t} v_{i}(t)}{\mu+(1-\mu) \frac{d}{d t} v_{i}(t) \cos \left(\theta_{i}(t)\right)} .
$$

Using the monotonicity with respect to $x$ of the function

$$
g(x)=\frac{(1-\mu) x}{\mu+(1-\mu) x \cos (\zeta)},
$$

and the inequality $\sin \left(\theta_{i}(t)\right) \leq \sin (\zeta)<\zeta, M^{-1} \leq \frac{d}{d t} v_{i}(t) \leq M$, we get

$$
\omega<\frac{(1-\mu) \sin \left(\theta_{i}(t)\right) \frac{d}{d t} v_{i}(t)}{\mu+(1-\mu) \frac{d}{d t} v_{i}(t) \cos \left(\theta_{i}(t)\right)}<\frac{(1-\mu) M \sin (\zeta)}{\mu+(1-\mu) M \cos (\zeta)} .
$$

Dividing both numerator and denominator by $(1-\mu) M$ and using the first part of (6.3) we have

$$
\bar{\theta}_{2 i}(t) \leq \omega<\frac{\zeta}{\frac{\mu}{(1-\mu) M}+\cos (\zeta)} \leq \frac{\zeta}{1+\lambda(\mu, \zeta)} .
$$

For the angle $\angle\left(\dot{\bar{C}}_{2 i-1}\left(v_{i}^{-1}(t)\right), \dot{\bar{C}}_{2 i}(t)\right)$ we define $\varpi=\angle\left(\dot{C}_{i}(t), \dot{\bar{C}}_{2 i}(t)\right)$.

Since $\varpi \leq \zeta$ and (3.1),

$$
\varpi=\angle\left(\dot{C}_{i}(t), \dot{\bar{C}}_{2 i}(t)\right) \leq \angle\left(\dot{C}_{i}(t), \dot{\bar{C}}_{2 i+1}(t)\right)=\omega<\theta_{i}(t)
$$

Now,

$$
\dot{\bar{C}}_{2 i}(t)=\alpha \dot{C}_{i}(t)+\beta u
$$

where $\left(\dot{C}_{i}(t), u\right)$ is an orthogonal basis in the spanned by $\dot{C}_{i}(t), \dot{C}_{i-1}\left(v_{i-1}^{-1}(t)\right)$, with

$$
\begin{gathered}
\alpha=\mathcal{K}\left((1-\mu)+\mu \frac{d}{d t} v_{i}(t) \cos \left(\theta_{i}(t)\right)\right. \\
\beta=\mathcal{K} \mu \frac{d}{d t} v_{i}(t) \sin \left(\theta_{i}(t)\right),
\end{gathered}
$$

where $\mathcal{K}$ is a positive number ensuring that $\left\|\dot{\bar{C}}_{2 i}(t)\right\|=1$.

In view of (A.3) we have

$$
\varpi=\arctan \left(\frac{(1-\mu) \frac{d}{d t} v_{i}(t) \sin \left(\theta_{i}(t)\right)}{(1-\mu)+\mu \frac{d}{d t} v_{i}(t) \cos \left(\theta_{i}(t)\right)}\right)
$$

By Taylor expansion,

$$
\arctan (\theta)<\theta, \quad 0<\theta<\frac{\pi}{4}
$$

which yields,

$$
\varpi \leq \frac{\mu \sin \left(\theta_{i}(t)\right) \frac{d}{d t} v_{i}(t)}{(1-\mu)+\mu \frac{d}{d t} v_{i}(t) \cos \left(\theta_{i}(t)\right)}
$$

Thus,

$$
\varpi<\frac{\left(\mu \sin \left(\theta_{i}(t)\right) \frac{d}{d t} v_{i}(t)\right.}{(1-\mu)+\mu \frac{d}{d t} v_{i}(t) \cos \left(\theta_{i}(t)\right)} .
$$

Using the monotonicity with respect to $x$ of the function

$$
g(x)=\frac{\mu x}{(1-\mu)+\mu x \cos (\zeta)},
$$


and the inequalities $\sin \left(\theta_{i}(t)\right) \leq \sin (\zeta)<\zeta, M^{-1} \leq \frac{d}{d t} v_{i}(t) \leq M$, we get

$$
\varpi<\frac{\left(\mu \sin \left(\theta_{i}(t)\right) \frac{d}{d t} v_{i}(t)\right.}{(1-\mu)+\mu \frac{d}{d t} v_{i}(t) \cos \left(\theta_{i}(t)\right)}<\frac{(\mu M \sin (\zeta)}{(1-\mu)+\mu M \cos (\zeta)} .
$$

Dividing both numerator and denominator by $\mu M$ and using the second part of (6.3) we have

$$
\varpi<\frac{\zeta}{\frac{1-\mu}{\mu M}+\cos (\zeta)} \leq \frac{\zeta}{2(1+\lambda(\mu, \zeta))} .
$$

Re-indexing the curves $i$ to $n-i$ for $i=0, \ldots, n-1$ we get

By the triangular inequality on the sphere we have

$$
\angle\left(\dot{C}_{i}(t), \dot{\bar{C}}_{2 i-1}\left(v_{i-1}^{-1}(t)\right)\right)<\frac{\zeta}{2(1+\lambda(\mu, \zeta))} .
$$

$$
\begin{aligned}
\angle\left(\dot{\bar{C}}_{2 i}(t), \dot{\bar{C}}_{2 i-1}\left(v_{i-1}^{-1}(t)\right)\right) \leq & \angle\left(\dot{C}_{i}(t), \dot{\bar{C}}_{2 i-1}\left(v_{i}^{-1}(t)\right)\right)+\angle\left(\dot{C}_{i}(t), \dot{\bar{C}}_{2 i}(t)\right) \\
& <\frac{\zeta}{1+\lambda(\mu, \zeta)} .
\end{aligned}
$$

Adding (A.2) and (A.4) completes the proof.

Recall that we assumed that $\zeta>0$, thereby, the inequalities are strict.

Proof of Lemma 6.5. Writing $\left\|\frac{d}{d t} \bar{C}_{2 i}(t)\right\|^{2}$ explicitly by (3.1), we get

By using

$$
\begin{aligned}
\left\|\frac{d}{d t} \bar{C}_{2 i}(t)\right\|^{2}=(1-\mu)^{2} & +2 \mu(1-\mu) \frac{d}{d t} v_{i}(t)\left\langle\dot{C}_{i}(t), \dot{C}_{i+1}\left(v_{i}(t)\right)\right\rangle \\
& +\left(\mu \frac{d}{d t} v_{i}(t)\right)^{2}
\end{aligned}
$$

one can deduce that

$$
\cos (\zeta) \leq\left\langle\dot{C}_{i}(t), \dot{C}_{i+1}\left(v_{i}(t)\right)\right\rangle \leq 1, \quad t \in I\left(C_{i}\right), \quad i=0,1, \ldots, n-1
$$

$$
\sqrt{\cos (\zeta)}\left(1-\mu+\mu \frac{d}{d t} v_{i}(t)\right) \leq\left\|\frac{d}{d t} \bar{C}_{2 i}(t)\right\| \leq 1-\mu+\mu \frac{d}{d t} v_{i}(t)
$$

The chain rule implies that $\frac{d}{d t} \bar{C}_{2 i}(t)=\dot{\bar{C}}_{2 i}\left(\tau_{2 i}(t)\right) \frac{d}{d t} \tau_{2 i}(t)$ which yields $\left\|\frac{d}{d t} \bar{C}_{2 i}(t)\right\|=\frac{d}{d t} \tau_{2 i}(t)$. Consequently, (6.5) follows. By similar arguments one can verify (6.6) and (6.7).

Proof of Theorem 6.6. By the definitions of $\mathbf{v}^{h}$ (Definition 3.1) and of the re-parametrizations $\tau_{2 i}$ and $\tau_{2 i+1}$ we get $v_{2 i}^{h}\left(\tau_{2 i}(t)\right)=$ $\tau_{2 i+1}\left(\tau_{2 i}(t)\right)$. The chain rule implies,

$$
\frac{d}{d \tau_{2 i}} v_{2 i}^{h}\left(\tau_{2 i}\right)=\frac{d}{d t} \tau_{2 i+1}\left(\tau_{2 i}^{-1}(t)\right) \frac{d}{d \tau_{2 i}} t\left(\tau_{2 i}\right), \quad i=0,1, \ldots, n-1 .
$$

According to Lemma 6.5 for $t=\tau_{2 i}^{-1}\left(\tau_{2 i}\right)$ one may verify that

$$
\frac{d}{d \tau_{2 i}} v_{2 i}^{h}\left(\tau_{2 i}\right) \leq \frac{f_{1}\left(\frac{d}{d t} v_{i}(t)\right)}{\sqrt{\cos (\zeta)}}
$$

where $f_{1}$ is defined in 8.2

Now by basic calculus we have

$$
f_{1}(1)=1, \quad \frac{d}{d x} f_{1}(1)=1-2 \mu, \quad \frac{d^{2}}{d x^{2}} f_{1}(1)<0
$$

which yields: $f_{1}(x)<1+(1-2 \mu)(x-1)$ for all $1<x$. Thus,

$$
\frac{d}{d \tau_{2 i}} v_{2 i}^{h}\left(\tau_{2 i}\right) \leq \frac{1+(1-2 \mu)(M-1)}{\sqrt{\cos (\zeta)}}
$$

Likewise by replacing $f_{1}$ by $f_{2}$ from 8.2 we get,

$$
\frac{d}{d \tau_{2 i+1}} v_{2 i+1}^{h}\left(\tau_{2 i+1}\right) \leq \frac{1+2 \mu(M-1)}{\sqrt{\cos (\zeta)}} .
$$

Taking the maximum over all possible $i$ and $\tau_{i}$

$$
\bar{M} \leq \frac{1+\mu^{a} \operatorname{st}(M-1)}{\sqrt{\cos (\zeta)}}
$$




\section{Acknowledgements}

We wish to express our gratitude to Professor Nira Dyn, who supervised this work along its various stages, for her substantial advice, involvement, and encouragement.

\section{REFERENCES}

[1] R. P. Agnew and R. J. Walker. A trigonometric infinite product. Amer. Math. Monthly, 54:206-211, 1947.

[2] Lars-Erik Andersson and Neil F. Stewart. Introduction to the mathematics of subdivision surfaces. Society for Industrial and Applied Mathematics (SIAM), Philadelphia, PA, 2010.

[3] G.M. Chaikin. An algorithm for high-speed curve generation. Comput. Graph. Image Process, 3:346-349, 1974.

[4] Erin Wolf Chambers, Éric Colin de Verdière, Jeff Erickson, Sylvain Lazard, Francis Lazarus, and Shripad Thite. Walking your dog in the woods in polynomial time. In Computational geometry (SCG'08), pages 101-109. ACM, New York, 2008.

[5] E. Cohen, R.F. Riesenfeld, and G. Elber. Geometric modeling with splines: an introduction, volume 1. AK Peters Wellesley, MA, 2001.

[6] Shmuel Cohen, Gershon Elber, and Reuven Bar-Yehuda. Matching of freeform curves. Computer-Aided Design, 29(5):369-378, 1997.

[7] C. Conti and N. Dyn. Blending based corner cutting subdivision scheme for nets of curves. Computer Aided Geometric Design, 27(4):340-358, 2010.

[8] C. de Boor. Cutting corners always works. Computer Aided Geometric Design, 4(1-2):125-131, 1987.

[9] C. De Boor. A practical guide to splines, volume 27. Springer Verlag, 2001.

[10] Manfredo P. do Carmo. Differential geometry of curves and surfaces. Prentice-Hall Inc., Englewood Cliffs, N.J., 1976. Translated from the Portuguese.

[11] Nira Dyn and David Levin. Subdivision schemes in geometric modelling. Acta Numer., 11:73-144, 2002.

[12] P. Grohs and J. Wallner. Log-exponential analogues of univariate subdivision schemes in lie groups and their smoothness properties. Approximation Theory XII: San Antonio, pages 181-190, 2007.

[13] U. Itai and N. Dyn. Generating surfaces by refinement of curves. Journal of Mathematical Analysis and Applications, 2011.

[14] J. Peters. C^1-surface splines. SIAM Journal on Numerical Analysis, 32(2):645-666, 1995.

[15] Jörg Peters and Ulrich Reif. Subdivision surfaces, volume 3 of Geometry and Computing. Springer-Verlag, Berlin, 2008. With introductory contributions by Nira Dyn and Malcolm Sabin.

[16] Bl. Sendov. Hausdorff approximations, volume 50 of Mathematics and its Applications (East European Series). Kluwer Academic Publishers Group, Dordrecht, 1990. Translated and revised from the Russian. 